\title{
Non-Equilibrium Quasiclassical Theory for Josephson Structures
}

\author{
M. H. S. Amin \\ D-Wave Systems Inc., 320-1985 West Broadway, Vancouver BC, V6J 4Y3, Canada
}

We present a non-equilibrium quasiclassical formalism suitable for studying linear response ac properties of Josephson junctions. The non-equilibrium self-consistency equations are satisfied, to very good accuracy, already in zeroth iteration. We use the formalism to study ac Josephson effect in a ballistic superconducting point contact. The real and imaginary parts of the ac linear conductance are calculated both analytically (at low frequencies) and numerically (at arbitrary frequency). They show strong temperature, frequency, and phase dependence. Many anomalous properties appear near $\phi=\pi$. We ascribe them to the presence of zero energy bound states.

\section{INTRODUCTION}

Quasiclassical theories are proven to be powerful for studying superconducting systems. They have been used by many researchers to study the properties of superconductors in the Meissner [1] or vortex states [2-4], as well as for surfaces $[5,6]$, point contacts $[7-10]$, or grain boundaries between different superconductors [11-14]. The equilibrium quasiclassical theory was developed by Eilenberger [15] and Larkin and Ovchinnikov [16] by integrating out irrelevant small scale degrees of freedom from the Nambu-Gorkov Green's function formulation of BCS superonductivity [17]. It was later generalized to nonequilibrium by Eliashberg [18] and Larkin and Ovchinnikov [19].

A major development in the numerical calculations in equilibrium quasiclassical theory was made after the introduction of Riccati-transformation by Schopohl and Maki $[2,20]$. The transformation changes the Eilenberger equations [15] into a set of decoupled non-linear differential equations, that can be integrated easily. In non-equilibrium, the presence of convolution integrals in the equations of motion for the Green's functions makes the formalism nontrivial. Nevertheless, a generalized version of the Schopohl-Maki transformations for nonequilibrium systems has been suggested by Eschrig et al. $[21,22]$.

Josephson junctions are important devices, not only due to their rich physical properties, but also for many applications, including sensitive magnetometers [23,24], ultrafast switching devices [25], qubit prototypes [26,27], etc. dc and ac properties of them have been the subject of extensive research [28-33]. Some of the investigations are based on the tunneling Hamiltonian approach [34], which provides a good approximation when the transparency of the junction is small (e.g. tunnel junctions). At large transparencies, which is the case for supercon- ducting point contacts or grain boundary junctions, multiple Andreev reflections (MAR) take place [35]. The MAR theories work well when the biasing voltage is large. At small biasing voltages, the number of Andreev reflections grows $(\sim \Delta / e V$, with $\Delta$ being the superconducting order parameter). Nevertheless, the formalism was applied to the case of single channel superconducting quantum point contact with small biasing voltage [31]. Alternatively, non-perturbative Hamiltonian method [10] and non-equilibrium Green's function method [33] were developed. However, most of these theories are suitable only when the applied voltage is constant. An exception is Ref. [32], which studies a superconducting quantum point contact in the presence of an ac voltage, but only in adiabatic regime. Thus a theory capable of studying high transparency Josephson junctions with an ac biasing voltage at arbitrary frequency is still lacking.

For equilibrium systems, the quasiclassical Green's functions theory has provided a convenient tool to study Josephson structures with arbitrary transparency and roughness of the junctions (see Ref. [13] and references therein). A generalization of the formalism to the nonequilibrium case, may also provide a powerful and convenient method to study ac properties of such structures. In this article, we rewrite the theory of Refs. [21,22] in a form suitable for studying properties of a general Josephson junction in the presence of an ac voltage at arbitrary frequency. To our knowledge, no such theory exists. We apply the theory to the case of a ballistic point contact between two conventional ( $s$-wave) superconductors. At low frequencies, we find closed analytical expressions for the real and imaginary parts of the ac conductivity. They agree very well with the numerical results, except where the low frequency expansion fails. Both quantities show strong temperature, frequency, and phase dependence. We observe anomalous behavior when the phase difference across the point contact approaches $\pi$. We relate that to the presence of the zero energy bound states.

Section II introduces the formalism and formulates it in a form appropriate for studying Josephson systems. Section III is devoted to calculation of ac current through a ballistic point contact between two $s$-wave superconductors. The low frequency analytical results are given in III-A. The numerical results, as well as a comparison with the analytical results, are presented in III-B. Section IV summarizes the main results. A detailed description of the theory and notations is given in two appendices. Understanding the appendices in great detail is not necessary for understanding the main body of the article and for application of the theory to other problems. 


\section{THE FORMALISM}

A convenient way to study non-equilibrium systems is to use Keldysh Green's functions [36]. The quasiclassical approximation of the Keldysh formalism introduces Retarded, Advanced, and Keldysh Green's functions: $\widehat{g}^{\alpha}$, $\alpha=R, A, K$. The first two describe spectral distribution of the states of the system, while the latter has information about population of those states. They are all $2 \times 2$ matrices, and functions of the Fermi velocity $\mathbf{v}_{F}$, quasiparticle energy $\epsilon$, position $\mathbf{r}$, and time $t$. The exact definitions of $\widehat{g}^{\alpha}$ and their equations of motion are given in appendix A [see Eqs. (A2) and (A6)]. The method we present here is a linear response treatment of $\widehat{g}^{\alpha}$. We consider a clean superconducting system in the absence of an external magnetic field. The coupling to the electromagnetic field is via the vector potential $\mathbf{A}$ and scalar potential $\Phi$. As will become soon clear, working with a gauge in which $\mathbf{A}=0$ simplifies the calculations significantly. In such a gauge, $\Phi$ is the only perturbation applied to the system, which we take to be small.

Let us first consider the case of a uniform (bulk) superconductor. We introduce a gauge transformation (see appendix A for details) $\Delta \mapsto \widetilde{\Delta}=e^{-i \delta \phi} \Delta$. Under such a transformation $e \Phi \mapsto e \Phi-(1 / 2) \partial_{t} \delta \phi$. (Throughout this article we use $\hbar=k_{B}=1$.) We choose $\delta \phi$ in such a way to exactly eliminate $\Phi$ from the gauge transformed dynamical equations [Eq. (A6)]. Thus, $\delta \phi$ should satisfy

$$
\frac{\partial \delta \phi}{\partial t}=2 e \Phi
$$

which is the well-known Josephson relation [37]. The vector potential $\mathbf{A}$ is still zero after this gauge transformation, because $\nabla \delta \phi=0$ within the bulk. The gauge transformed equation of motion is therefore exactly the same as the equilibrium equation. As a result, $\widetilde{\Delta}=\Delta_{0}$, or $\Delta=\Delta_{0} e^{i \delta \phi}$, where $\Delta_{0}$ is the equilibrium order parameter. This means that the magnitude of the order parameter, $|\Delta|$, is independent of $\Phi$, whereas its phase varies with $\Phi$ via the Josephson relation (1). In other words, if the only perturbation to the system is through the time varying potential $\Phi$, its only effect is to change the phase of the order parameter [38].

The above simple observation has very important consequence in our linear response formalism. One can write the original (gauge dependent) order parameter as

$$
\Delta=\Delta_{0} e^{i \delta \phi} \approx \Delta_{0}(1+i \delta \phi)=\Delta_{0}+\delta \Delta,
$$

where $\delta \Delta$ is the non-equilibrium linear response correction to the order parameter. In Fourier space, the Josephson relation (1) becomes $\delta \phi=i 2 e \Phi(\omega) / \omega$, which yields

$$
\delta \Delta(\omega)=-\frac{2 \Delta_{0}}{\omega} e \Phi(\omega),
$$

This value indeed satisfies the self-consistency equation for the homogeneous superconductor [cf. Eq. (23)]. To ensure small perturbation requirement, one needs $e \Phi \ll$ $\omega$. Charge neutrality condition in the bulk is also satisfied automatically by using (3). This can be seen simply from the above gauge transformation argument, taking into account the neutrality of the unperturbed (equilibrium) system.

We now consider a Josephson junction with an equilibrium phase difference $\phi$ and an ac voltage $V=V_{0} \cos \omega t$ across the junction. Far away from the junction, we take the phase of the order parameter and the scalar potential on the left $(\mathrm{L})$ and right $(\mathrm{R})$ sides to be $\phi_{\mathrm{R}, \mathrm{L}}= \pm \phi / 2$ and $\Phi_{\mathrm{R}, \mathrm{L}}= \pm\left(V_{0} / 2\right) \cos \omega t$, respectively. In general, the phase of the order parameter is space dependent. Therefore, performing the above gauge transformation will produce a vector potential $\mathbf{A}=(c / 2 e) \nabla \delta \phi\left(\propto j_{\mathrm{ac}} / j_{\mathrm{c}, \text { bulk }}\right.$, where $j_{\mathrm{ac}}$ is the ac current density in the banks and $j_{\text {c,bulk }}$ is the bulk critical current density), which invalidates our arguments. However, in most practical systems, $j_{\text {ac }} \ll j_{\mathrm{c}} \ll j_{\text {c, bulk, }}$, where $j_{\mathrm{c}}$ is the Josephson current density. The corrections to Eq. (3) are therefore small and $O\left(j_{\mathrm{c}} / j_{\text {c,bulk }}\right)$ [39]. Thus one can still use (3), as a very good approximation, even when $\Delta_{0}$ is space dependent. This removes the necessity for an iterative procedure (the main obstacle in these types of calculations) in order to self-consistently calculate $\delta \Delta$, and satisfy charge neutrality condition (within the bulk) [21,22]. The equilibrium order parameter $\Delta_{0}$, however, should be calculated self-consistently using the common iterative methods; convergence of such calculations is proven to be very good, especially when using the Matsubara technique [13].

\section{A. Equilibrium Solution}

In equilibrium, the retarded and advanced Green's functions can be written in terms of the Riccati amplitudes $a_{0}^{\alpha}$ and $b_{0}^{\alpha}$ in a way very similar to the conventional method for the Matsubara Green's functions [13].

$$
g_{0}^{\alpha}=s^{\alpha} \frac{1-a_{0}^{\alpha} b_{0}^{\alpha}}{1+a_{0}^{\alpha} b_{0}^{\alpha}}, \quad f_{0}^{\alpha}=s^{\alpha} \frac{2 a_{0}^{\alpha}}{1+a_{0}^{\alpha} b_{0}^{\alpha}},
$$

where

$$
s^{\alpha}=\left\{\begin{array}{ll}
+ & \text { for } \alpha=R \\
- & \text { for } \alpha=A
\end{array} .\right.
$$

The subscript "0" denotes equilibrium quantities. The Riccati amplitudes satisfy the following Riccati equations [41]

$$
\begin{aligned}
\mathbf{v}_{F} \cdot \nabla a_{0}^{\alpha} & =2 i \epsilon^{\alpha} a_{0}^{\alpha}-\left(a_{0}^{\alpha}\right)^{2} \Delta_{0}^{*}+\Delta_{0}, \\
-\mathbf{v}_{F} \cdot \nabla b_{0}^{\alpha} & =2 i \epsilon^{\alpha} b_{0}^{\alpha}-\left(b_{0}^{\alpha}\right)^{2} \Delta_{0}+\Delta_{0}^{*},
\end{aligned}
$$

where $\epsilon^{\alpha}=\epsilon+i s^{\alpha} \eta$, with $\epsilon$ and $\eta$ being the real and imaginary parts of the quasiparticle energy respectively. $\eta$ is the quasiparticle damping, related to the inelastic lifetime $\tau$ of the quasiparticles by $\eta=1 / \tau$ [40]. Notice 
that the scalar potential $\Phi$ does not appear in the equilibrium equations. The boundary conditions are the bulk solutions of Eq. (6):

$$
a_{0}^{\alpha}=\frac{\Delta_{0}}{-i \epsilon^{\alpha}+s^{\alpha} \Omega^{\alpha}}, \quad b_{0}^{\alpha}=\frac{\Delta_{0}^{*}}{-i \epsilon^{\alpha}+s^{\alpha} \Omega^{\alpha}},
$$

where $\Omega^{\alpha}=\sqrt{\left|\Delta_{0}\right|^{2}-\left(\epsilon^{\alpha}\right)^{2}}$. The differential equations (6) and the boundary conditions (7) can be obtained from their counterparts in Matsubara formalism (see Ref. [13] for example), by changing $\omega_{n} \rightarrow-i \epsilon^{\alpha}$, where $\omega_{n}$ is the Matsubara frequency.

To calculate the Riccati amplitudes at other points, one should define quasiclassical trajectories as straight lines in the direction of $\mathbf{v}_{F}$ (see Fig. 1). $a_{0}^{R}$ and $b_{0}^{A}$ are obtained by integrating (6) in the direction of $\mathbf{v}_{F}$ along the trajectory, starting from the boundary conditions $(7)$ at $-\infty$. For $b_{0}^{R}$ and $a_{0}^{A}$, integrations are taken in the opposite direction. The following symmetries exist for the equilibrium functions

$$
a_{0}^{A}=-\left(b_{0}^{R}\right)^{*}, \quad b_{0}^{A}=-\left(a_{0}^{R}\right)^{*} .
$$

It is therefore sufficient to calculate one of the sets of retarded or advanced functions.

In equilibrium, the Keldysh Green's function is related to the retarded and advanced ones by

$$
\widehat{g}_{0}^{K}=\left(\widehat{g}_{0}^{R}-\widehat{g}_{0}^{A}\right) \mathcal{F}
$$

where

$$
\mathcal{F} \equiv \tanh \left(\frac{\epsilon}{2 T}\right)
$$

takes into account the thermal distribution of the quasiparticles. Equilibrium current and charge densities are calculated by Eqs. (A17) and (A18), summing over all trajectories.

\section{B. Linear Response Solution}

Generalization of the above Riccati-transformation to non-equilibrium is discussed in appendix B. The presence of the $\otimes$-operations [see Eq. (A8) for definition] makes calculations nontrivial. However, significant simplification arises when one side of the $\otimes$-operation is an equilibrium quantity (and therefore time/frequency independent). More specifically, in frequency space we have

$$
\begin{aligned}
& P_{0}(\epsilon) \otimes Q(\epsilon, \omega)=P_{0}\left(\epsilon+\frac{\omega}{2}\right) Q(\epsilon, \omega), \\
& P(\epsilon, \omega) \otimes Q_{0}(\epsilon)=P(\epsilon, \omega) Q_{0}\left(\epsilon-\frac{\omega}{2}\right) .
\end{aligned}
$$

To take the advantage of this property, we use linear expansion. In other words, we assume that the perturbation to the system (i.e. $\Phi$ ) is so small that the linear expansion of the Green's functions provides a good approximation to the exact solution. All the differential equations [Eqs. (B2) and (B4)] will then be linear in the time-varying parts, and Fourier transformation will be straightforward: no complicated convolution integrals arise.

Let us introduce simplifying notation

$$
\epsilon_{ \pm}=\epsilon \pm \frac{\omega}{2}, \quad Q_{0 \pm}=Q_{0}\left(\epsilon_{ \pm}\right)
$$

We define linear response Green's functions as $\delta \widehat{g}^{\alpha}=$ $\widehat{g}^{\alpha}-\widehat{g}_{0}^{\alpha}$, where $\widehat{g}_{0}^{\alpha}$ are the equilibrium Green's functions. Similarly, we introduce small corrections to the Riccati amplitudes $\delta a^{\alpha}=a^{\alpha}-a_{0}^{\alpha}$ and $\delta b^{\alpha}=b^{\alpha}-b_{0}^{\alpha}$. The linear response Green's functions are then given in terms of the Riccati amplitudes by

$$
\begin{aligned}
& \delta g^{\alpha}=-2 s^{\alpha} \frac{\delta a^{\alpha} b_{0-}^{\alpha}+\delta b^{\alpha} a_{0+}^{\alpha}}{\left(1+a_{0+}^{\alpha} b_{0+}^{\alpha}\right)\left(1+a_{0-}^{\alpha} b_{0-}^{\alpha}\right)}, \\
& \delta f^{\alpha}=2 s^{\alpha} \frac{\delta a^{\alpha}-\delta b^{\alpha} a_{0+}^{\alpha} a_{0-}^{\alpha}}{\left(1+a_{0+}^{\alpha} b_{0+}^{\alpha}\right)\left(1+a_{0-}^{\alpha} b_{0-}^{\alpha}\right)},
\end{aligned}
$$

for $\alpha=R, A$.

We also define an anomalous Green's function $\delta \widehat{g}^{X}$ by

$$
\delta \widehat{g}^{K}=\delta \widehat{g}^{X}\left(\mathcal{F}_{+}-\mathcal{F}_{-}\right)+\delta \widehat{g}^{R} \mathcal{F}_{-}-\delta \widehat{g}^{A} \mathcal{F}_{+} .
$$

Correspondingly, we introduce anomalous functions $\delta a^{X}$ and $\delta b^{X}$ [see Eqs. (B9) and (B10)] which are related to the Green's functions through

$$
\begin{aligned}
\delta g^{X} & =2 \frac{\delta a^{X}-\delta b^{X} a_{0+}^{R} b_{0-}^{A}}{\left(1+a_{0+}^{R} b_{0+}^{R}\right)\left(1+a_{0-}^{A} b_{0-}^{A}\right)}, \\
\delta f^{X} & =2 \frac{\delta a^{X} a_{0-}^{A}+\delta b^{X} a_{0+}^{R}}{\left(1+a_{0+}^{R} b_{0+}^{R}\right)\left(1+a_{0-}^{A} b_{0-}^{A}\right)} .
\end{aligned}
$$

The differential equations describing $\delta a^{\alpha}$ and $\delta b^{\alpha}(\alpha=$ $R, A, X)$ have general forms

$$
\begin{aligned}
\mathbf{v}_{F} \cdot \nabla \delta a^{\alpha} & =A^{\alpha} \delta a^{\alpha}+B^{\alpha}, \\
-\mathbf{v}_{F} \cdot \nabla \delta b^{\alpha} & =\widetilde{A}^{\alpha} \delta b^{\alpha}+\widetilde{B}^{\alpha},
\end{aligned}
$$

with $A$ 's and $B$ 's given by [41]

$$
\begin{aligned}
& A^{\alpha}=2 i \epsilon-\Delta_{0}^{*}\left(a_{0+}^{\alpha}+a_{0-}^{\alpha}\right) \\
& B^{\alpha}=\delta \Delta+a_{0+}^{\alpha} a_{0-}^{\alpha} \delta \Delta^{*}-i e \Phi\left(a_{0+}^{\alpha}-a_{0-}^{\alpha}\right) \\
& \widetilde{A}^{\alpha}=2 i \epsilon-\Delta_{0}\left(b_{0+}^{\alpha}+b_{0-}^{\alpha}\right) \\
& \widetilde{B}^{\alpha}=-\delta \Delta^{*}-b_{0+}^{\alpha} b_{0-}^{\alpha} \delta \Delta+i e \Phi\left(b_{0+}^{\alpha}-b_{0-}^{\alpha}\right)
\end{aligned}
$$

for the retarded $(\alpha=R)$ and advanced ( $\alpha=A)$ functions, and

$$
\begin{aligned}
& A^{X}=i \omega-a_{0+}^{R} \Delta_{0}^{*}+b_{0-}^{A} \Delta_{0}, \\
& B^{X}=-a_{0+}^{R} \delta \Delta^{*}+b_{0-}^{A} \delta \Delta-i e \Phi\left(1+a_{0+}^{R} b_{0-}^{A}\right),
\end{aligned}
$$




$$
\begin{aligned}
& \widetilde{A}^{X}=i \omega-b_{0+}^{R} \Delta_{0}+a_{0-}^{A} \Delta_{0}^{*}, \\
& \widetilde{B}^{X}=b_{0+}^{R} \delta \Delta-a_{0-}^{A} \delta \Delta^{*}+i e \Phi\left(1+b_{0+}^{R} a_{0-}^{A}\right),
\end{aligned}
$$

for the anomalous ones $(\alpha=X) . \delta \Delta \equiv \Delta-\Delta_{0}$, is given by Eq. (3) and satisfies the self-consistency equation

$$
\delta \Delta\left(\mathbf{v}_{F}\right)=\frac{N_{F}}{4 i} \int_{-\epsilon_{c}}^{\epsilon_{c}} d \epsilon\left\langle V\left(\mathbf{v}_{F}, \mathbf{v}_{F}^{\prime}\right) \delta f^{K}\left(\mathbf{v}_{F}^{\prime}\right)\right\rangle_{\mathbf{v}_{F}^{\prime}},
$$

where $V\left(\mathbf{v}_{F}, \mathbf{v}_{F}^{\prime}\right)$ is the interaction potential, $N_{F}$ the density of states at the Fermi surface, and $\epsilon_{c}$ the energy cutoff. The bulk boundary conditions for the amplitudes are

$$
\delta a^{\alpha}=-\frac{B^{\alpha}}{A^{\alpha}}, \quad \delta b^{\alpha}=-\frac{\widetilde{B}^{\alpha}}{\widetilde{A}^{\alpha}} .
$$

To assure stability of the integrations, Eqs. (18) should be integrated along the trajectory in the direction of $\mathbf{v}_{F}$ for $\delta a^{R}, \delta b^{A}$, and $\delta a^{X}$, but in the opposite direction for $\delta b^{R}$, $\delta a^{A}$, and $\delta b^{X}$ (see Fig. 1). Having the Green's functions, the non-equilibrium correction to the current density is given by

$$
\delta \mathbf{j}=\frac{e N_{F}}{4} \int_{-\epsilon_{c}}^{\epsilon_{c}} d \epsilon\left\langle\mathbf{v}_{F} \operatorname{Tr}\left[\widehat{\tau}_{3} \delta \widehat{g}^{K}\right]\right\rangle,
$$

and the charge density is found from

$$
\delta \rho=e N_{F}\left(-2 e \Phi+\frac{1}{4} \int_{-\epsilon_{c}}^{\epsilon_{c}} d \epsilon\left\langle\operatorname{Tr}\left[\delta \widehat{g}^{K}\right]\right\rangle\right) .
$$

\section{AC JOSEPHSON EFFECT IN A SUPERCONDUCTING POINT CONTACT}

A ballistic superconducting point contact is probably the simplest system that the method described here can be applied to. It brings extra simplicity because even the equilibrium solutions can be found non-self-consistently [7]. Nevertheless, the system shows rich and nontrivial physical behavior. Let us consider an orifice between two conventional ( $s$-wave) superconductors (Fig. 1), dimension of which much smaller than the inelastic scattering length and coherence length of the superconductors $\left(d \ll l_{\tau}=v_{F} \tau, \xi_{0}=v_{F} / \pi\left|\Delta_{0}\right|\right)$. We assume perfect transparency at the contact, although generalization to arbitrary transparency is straightforward [13,22]. We take the equilibrium order parameter to be constant on both sides of the contact, $\Delta_{\mathrm{L}, \mathrm{R}}=\left|\Delta_{0}\right| e^{i \phi_{\mathrm{L}, \mathrm{R}}}$, with $\phi_{\mathrm{L}, \mathrm{R}}= \pm \phi / 2$, where $\phi$ is the phase difference between the two sides. Here, L (R) denotes the left (right) side of the contact.

We also take $\delta \Delta$ to be constant on each side of the contact, given by Eq. (3). The scalar potential $\Phi$ is taken to be $\Phi_{\mathrm{L}, \mathrm{R}}= \pm\left(V_{0} / 2\right) \cos \omega t$ on the left and right sides respectively, $V_{0}$ being the amplitude of the potential difference. It is taken to be a real number and so small

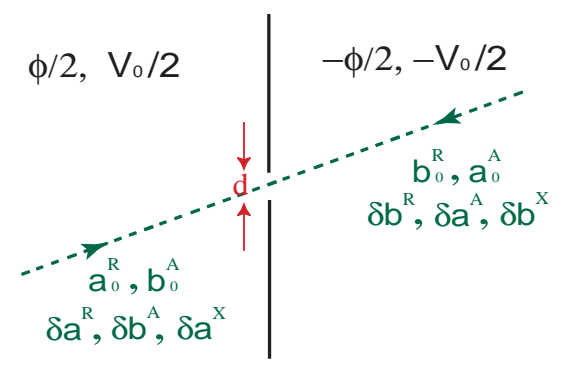

FIG. 1. Two superconducting regions connected via an orifice. The dashed line shows the quasiclassical trajectory. The arrows indicate the directions of integration.

that the linear expansion provides a good approximation $\left(e V_{0} \ll\left|\Delta_{0}\right|, \omega\right)$.

To find the current response, $I$, of the system, we calculate the current density $\delta j_{z}$ at the orifice, using Eq. (25), and then integrate it over the area $S$ of the orifice. In Fourier space, $I(\omega)$ can be a complex number. The real part of it describes the dissipation of the system, while its imaginary part gives information about inductive or capacitive behavior of the system. The linear admittance of the system is defined by $Y(\omega)=I(\omega) / V_{0}(=1 / Z$, where $Z$ is the impedance of the contact).

We proceed with the calculation of the current in two different ways. First, we find analytical results in the regime of small $\omega$ and $\eta$. We then provide the results of full numerical calculation and compare them with the analytical ones.

\section{A. Low Energy Analytical Results}

In the case of a point contact, it is not difficult to obtain analytical results. Since the superconductor is homogeneous everywhere except near the contact, the solution to the functions $a$ and $b$ at the contact is almost equal to their bulk values. More specifically, $a^{R}(0)=a^{R}(-\infty)$, $b^{R}(0)=b^{R}(+\infty)$, etc. From now on we drop the arguments and just write $a^{R}, b^{R}$, etc., keeping in mind that what we mean is the values at the position of the contact. Substituting these values in the corresponding equations, one can obtain analytical expressions for the current. The exact expression is rather complicated and does not give any more insight than the numerical results. It, however, can be significantly simplified in low energy regime.

Here, we calculate the contact admittance in the regime $\eta, \omega \ll\left|\Delta_{0}\right|, T$ (but of course $\omega \gg e V_{0}$ ). Let us first introduce the following parameterization

$$
\epsilon^{\alpha}=\left|\Delta_{0}\right| \cos \gamma^{\alpha}, \quad \alpha=R, A
$$

where $\gamma^{\alpha}$ is a complex number. We therefore find $\Omega^{\alpha}=$ $\left|\Delta_{0}\right| \sin \gamma^{\alpha}$. This choice of notation significantly simplifies the form of $a_{0}$ and $b_{0}$. For instance from (7), taking 
the phase of the order parameter to be $+\phi / 2$ (left side of the contact), one finds

$$
a_{0}^{R}=\frac{\left|\Delta_{0}\right| e^{i \phi / 2}}{-i \epsilon^{R}+\Omega^{R}}=i e^{i\left(\phi / 2-\gamma^{R}\right)}=i e^{-i \delta \gamma^{R}},
$$

where $\delta \gamma^{R} \equiv \gamma^{R}-\phi / 2$. Similarly, $b_{0}^{R}$ has to be calculated on the right side of the contact (where the phase is $-\phi / 2$ ) and turns out to be exactly equal to $a_{0}^{R}$. In general, one can write

$$
b_{0}^{\alpha}=a_{0}^{\alpha}=i e^{-i s^{\alpha} \delta \gamma^{\alpha}} .
$$

First notice that $1+a_{ \pm}^{\alpha} b_{ \pm}^{\alpha}=1-e^{-2 i s^{\alpha} \delta \gamma_{ \pm}^{\alpha}}$ vanishes as $\delta \gamma_{ \pm}^{\alpha} \equiv \delta \gamma^{\alpha}\left(\epsilon_{ \pm}^{\alpha}\right) \rightarrow 0$ (or as $\gamma_{ \pm}^{\alpha} \rightarrow \phi / 2$ ). Because of such expressions in the denominators of Eqs. (13) and (16), the most important contribution to those equations must come from points close to $\epsilon=\epsilon_{0}(\phi)$ for which $\delta \gamma^{\alpha} \ll 1$. Here

$$
\epsilon_{0}(\phi)=\left|\Delta_{0}\right| \cos \frac{\phi}{2}
$$

( $\pm \epsilon_{0}$ are indeed the energies of the Andreev bound states in the contact [36].) We can therefore focus only on those points. Expanding the numerators of (13) and (16) up to first order in $\delta \gamma^{\alpha}$, (i.e. first order in $\omega$ and $\eta$ ), we find

$$
\begin{gathered}
\delta g^{R}=\frac{e V}{\omega}\left[\frac{1}{\delta \gamma_{+}^{R} \delta \gamma_{-}^{R}}+\left(\frac{\epsilon_{0}}{2 \Omega_{0}}-i\right)\left(\frac{1}{\delta \gamma_{+}^{R}}-\frac{1}{\delta \gamma_{-}^{R}}\right)\right] \\
\delta g^{A}=\frac{e V}{\omega}\left[\frac{1}{\delta \gamma_{+}^{A} \delta \gamma_{-}^{A}}+\left(\frac{\epsilon_{0}}{2 \Omega_{0}}+i\right)\left(\frac{1}{\delta \gamma_{+}^{A}}-\frac{1}{\delta \gamma_{-}^{A}}\right)\right] \\
\delta g^{X}=\frac{e V}{\omega}\left[\frac{1}{\delta \gamma_{+}^{R} \delta \gamma_{-}^{A}}+\left(\frac{\epsilon_{0}}{2 \Omega_{0}}+i\right) \frac{1}{\delta \gamma_{+}^{R}}\right. \\
\left.+\left(\frac{\epsilon_{0}}{2 \Omega_{0}}-i\right) \frac{1}{\delta \gamma_{-}^{A}}\right],
\end{gathered}
$$

where

$$
\Omega_{0}(\phi)=\left|\Delta_{0}\right| \sin \frac{\phi}{2} .
$$

Let us write $\epsilon=\epsilon_{0}+\epsilon^{\prime}\left(\epsilon^{\prime} \ll\left|\Delta_{0}\right|\right)$. For $\phi \neq 0$ (we will discuss the $\phi=0$ case later), one can write

$$
\delta \gamma_{ \pm}^{\alpha} \approx-\frac{1}{\Omega_{0}}\left(\epsilon^{\prime}+i s^{\alpha} \eta \pm \frac{\omega}{2}\right) .
$$

Therefore

$$
\frac{1}{\delta \gamma_{ \pm}^{\alpha}} \approx-\Omega_{0}\left[\frac{P}{\epsilon^{\prime} \pm \omega / 2}-i \pi s^{\alpha} \delta\left(\epsilon^{\prime} \pm \frac{\omega}{2}\right)\right],
$$

where $P$ gives the principal value integral when integrating over $\epsilon^{\prime}$. Because of symmetry, the principle value integrals are negligible after integration. We therefore write

$$
\frac{1}{\delta \gamma_{ \pm}^{\alpha}} \approx i \pi s^{\alpha} \Omega_{0} \delta\left(\epsilon^{\prime} \pm \frac{\omega}{2}\right)
$$

On the other hand

$$
\begin{aligned}
\frac{1}{\delta \gamma_{+}^{\alpha} \delta \gamma_{-}^{\alpha}} & =\frac{\Omega_{0}^{2}}{\omega}\left(\frac{1}{\epsilon^{\prime}-\omega / 2+i s^{\alpha} \eta}-\frac{1}{\epsilon^{\prime}+\omega / 2+i s^{\alpha} \eta}\right) \\
& \approx \frac{i \pi s^{\alpha} \Omega_{0}^{2}}{\omega}\left[\delta\left(\epsilon^{\prime}+\frac{\omega}{2}\right)-\delta\left(\epsilon^{\prime}-\frac{\omega}{2}\right)\right] .
\end{aligned}
$$

Similarly

$$
\begin{aligned}
\frac{1}{\delta \gamma_{+}^{R} \delta \gamma_{-}^{A}} & =\frac{\Omega_{0}^{2}}{\omega+2 i \eta}\left(\frac{1}{\epsilon^{\prime}-\omega / 2-i \eta}-\frac{1}{\epsilon^{\prime}+\omega / 2+i \eta}\right) \\
& \approx \frac{i \pi \Omega_{0}^{2}}{\omega+2 i \eta}\left[\delta\left(\epsilon^{\prime}+\frac{\omega}{2}\right)+\delta\left(\epsilon^{\prime}-\frac{\omega}{2}\right)\right] .
\end{aligned}
$$

Substituting (35)-(37) into (31) and using (15), one can calculate $\delta g^{K}$ and thereby the total current using (25). Taking the integral over $\epsilon^{\prime}$ in (25), expanding the resulting hyperbolic tangents around $\epsilon_{0}$ (to the first order in $\omega / T \ll 1)$ and keeping only the leading order terms, we find the admittance to be

$$
\begin{aligned}
Y(\omega)= & \frac{\pi}{\omega R_{N}}\left[\frac{\Omega_{0}^{2}}{2 T}\left(\frac{2 \eta}{\omega+2 i \eta}\right) \operatorname{sech}^{2} \frac{\epsilon_{0}}{2 T}\right. \\
& \left.+i \epsilon_{0} \tanh \frac{\epsilon_{0}}{2 T}\right],
\end{aligned}
$$

where $R_{N}=2 / e^{2} v_{F} N_{F} S$ is the normal (Sharvin) resistance of the point contact. Equation (38) agrees with the result obtained by Averin and Bardas [32] in adiabatic regime [42]. The quasiparticle conductance is given by the real part of (38):

$$
\frac{G(\omega)}{G_{N}}=\frac{\pi \eta \Omega_{0}^{2}}{\left(\omega^{2}+4 \eta^{2}\right) T} \operatorname{sech}^{2} \frac{\epsilon_{0}}{2 T},
$$

where $G_{N}=1 / R_{N}$. Notice that the right hand side of Eq. (39) vanishes at $\phi=0$. This however is the point at which the linear expansion (33) fails. One therefore expects the terms neglected in (39) to dominate $G(\phi=$ $0)$. Similarly, at small $T$, Eq. (39) is exponentially small (except at $\phi=\pi$ ). Since in such a regime, $\omega / T$ becomes large, the expansion of the hyperbolic tangents in powers of $\omega / T$ will be invalid. Therefore, deviation from (39) at low $T$ is expected. In the next subsection, we will see such deviations in the numerical results.

The imaginary part of the admittance is also important because it provides information about the inductive or capacitive behavior of the junction. Notice that at $\phi=0$, the first term in (38) vanishes but the second term survives. Thus unlike the conductance, $\operatorname{Im}(Y)$ does not vanish; it rather stays finite and behaves purely inductively $(\sim 1 / \omega L)$. Similarly, near $T=0$, the second term in (38) dominates, resulting in a finite (again inductive) $\operatorname{Im}(Y)$. One therefore expects that the leading order expansion provides good approximation. The exception is at $\phi=\pi$ where $\epsilon_{0}=0$ and thus the second term in (38) vanishes. The higher order terms therefore play important role in such a case. In the next subsection, we observe this behavior by comparing with the numerical results. 


\section{B. Numerical Results}

We now present the results of our numerical calculation. The value of $\left|\Delta_{0}\right|$ is calculated directly from the BCS gap equation [34]:

$$
1=\lambda \int_{\left|\Delta_{0}\right|}^{\epsilon_{c}} \frac{d \epsilon}{\sqrt{\epsilon^{2}-\left|\Delta_{0}\right|^{2}}} \tanh \left(\frac{\epsilon}{2 T}\right)
$$

where the dimensionless coupling constant $\lambda$ is chosen in such a way to give $\Delta_{0} \rightarrow 0$ as $T \rightarrow T_{c}$. Near $T=0$, one finds $\left|\Delta_{0}\right| \approx 1.75 T_{c}$. All the energy scales $\left(T, \epsilon, \omega, e V_{0}, \eta\right.$, etc.) are normalized to $T_{c}$ and $Y(\omega)$ to $G_{N}$. In all calculations, we take $\eta=0.01$ and $\epsilon_{c}=20$.

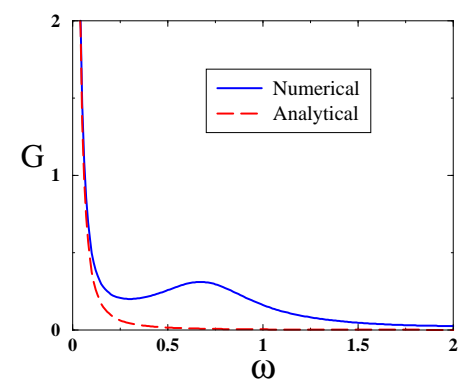

FIG. 2. Linear conductance $G$ (normalized to $G_{N}$ ) as a function of $\omega$, for $\phi=3 \pi / 4$ at $T=0.1$. ( $\omega$ and $T$ as well as other energy scales are normalized to $T_{c}$.)

Fig. 2 compares the result of numerical calculation of $G$ at $\phi=3 \pi / 4$ with the analytical result obtained from Eq. (39). As expected, the two curves overlap at low frequencies but deviate at larger $\omega$. Around $\omega=0$, there exists a sharp peak corresponding to the Lorentzian $\omega$ dependence in (39). At larger frequencies, a second peak appears in the numerical curve which is absent in the analytical one. The peak clearly results form the higher order contributions which were neglected in derivation of (38). Fig. 3 displays numerical $G-\omega$ curves at different phase differences. At $\phi=\pi$ (Fig. 3d), the sharp peak at $\omega=0$ has the largest value. At smaller phase differences, this peak becomes less pronounced and eventually disappears at $\phi=0$, as it should according to (39); the equation actually predicts zero conductance, therefore the small conductance in Fig. 3a is completely due to the terms neglected in (39). The second peak, however, appears at $\omega=\left|\Delta_{0}\right| \approx 1.75$ for $\phi=0$ (Fig. 3a), and moves towards $\omega=0$ as $\phi \rightarrow \pi$. It is easy to see that the peak is always at $\omega=\epsilon_{0}(\phi)$, i.e., the energy of the Andreev levels.

Fig. 4 displays the conductance $G$ as a function of the phase difference across the contact for different frequencies. It is clear from the figure that $G$ is strongly phasedependent. Especially, it is sharply peaked close to $\phi=\pi$ [for $\omega=0.01$, it is more than five orders of magnitude larger than $G(\phi=0)$, see Fig. $4 \mathrm{~b}]$. The strong conductance can be attributed to the existence of zero energy
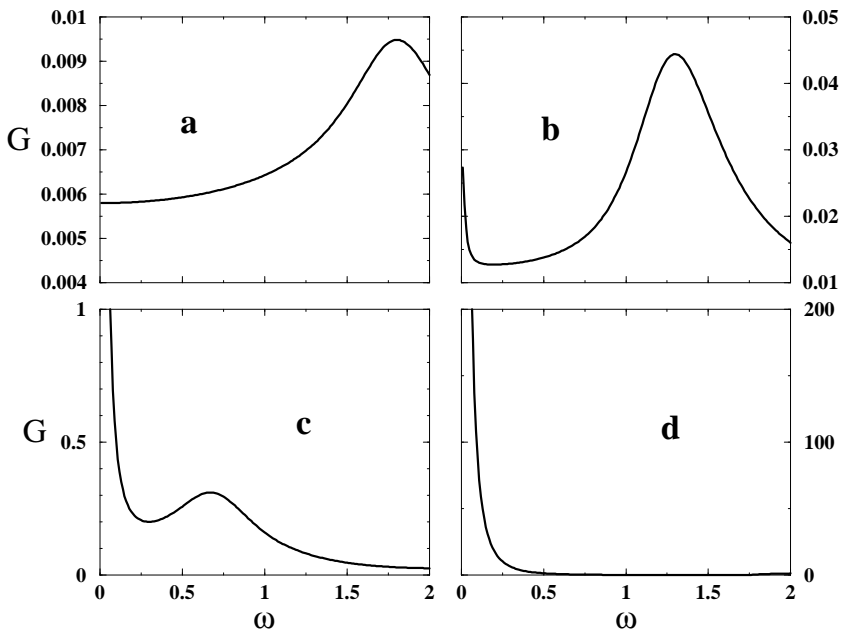

FIG. 3. Linear conductance as a function of $\omega$ at $T=0.1$. The phase differences are (a) $\phi=0$, (b) $\phi=\pi / 2$, (c) $\phi=3 \pi / 4$, and (d) $\phi=\pi$. (a)

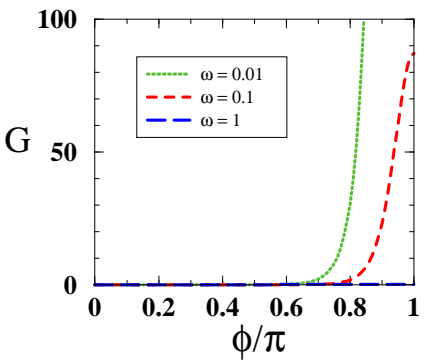

(b)

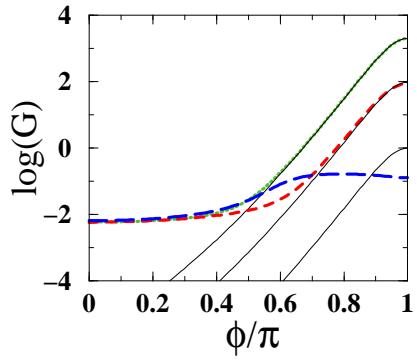

FIG. 4. (a) Linear conductance $G$ as a function of the phase difference at $T=0.1$, for different frequencies $\omega$. (b) The same data on logarithmic scale. The solid lines correspond to the analytical results. Here (and in the figures that follow), the legend is common between (a) and (b).

Andreev bound states (ZBS) [i.e. $\epsilon_{0}(\phi=\pi)=0$ ]; they provide large density of states at zero energy. A comparison with the analytical results is shown, in logarithmic scale, in Fig. 4b. As expected, the agreement between the two calculations at $\omega=0.01$, is good near $\phi=\pi$ but they deviate as $\phi \rightarrow 0$. The curves however overlap less at higher frequencies. Especially, at $\omega=1$ they show completely different phase dependence.

The temperature dependence of the linear conductance is presented in Fig. 5a. All the curves join at $G=1$ (or $G=G_{N}$ before normalization) as $T \rightarrow T_{c}$. This indeed is expected, because at $T=T_{c}$ the superconductor becomes normal. It is clear from the figure that the conductance behaves completely differently at $\phi=\pi$ compared to other phase differences. At $\phi=\pi$, the conductance grows with lowering the temperature and exhibits a $1 / T$ dependence in agreement with (39). This type of $1 / T$ behavior also exists in the dc Josephson current at $\phi=\pi$, 
(a)
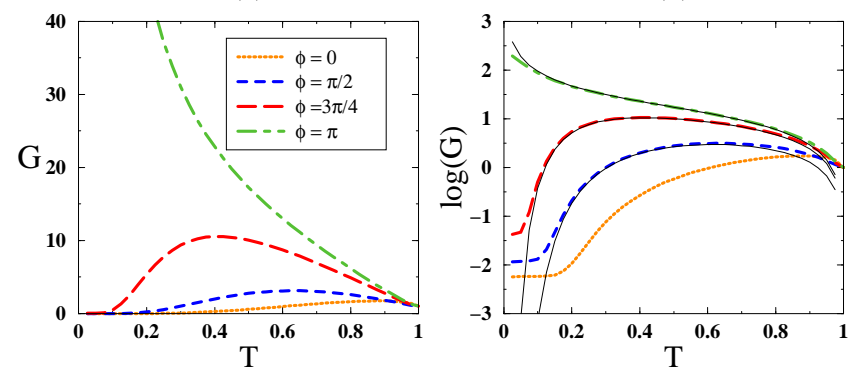

FIG. 5. (a) Linear conductance as a function of temperature for different phase differences at $\omega=0.1$. (b) The same data plotted in logarithmic scale. Solid lines are analytical results. Notice that at $\phi=0$, Eq. (39) gives $G=0$, therefore no analytical curve is shown in the figure. (a)

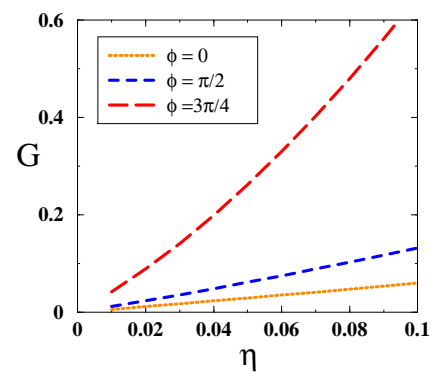

(b)

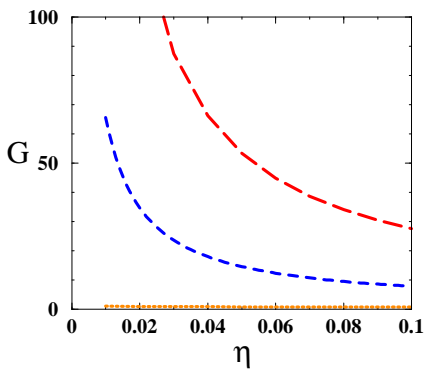

FIG. 6. (a) Conductance $G$ as a function of damping rate $\eta$ at $\omega=0.005$. (a) $T=0.01$, (b) $T=0.5$.

and is associated with the current carried by the ZBS. For $\phi=0, \pi / 2$, and $3 \pi / 4$, and at intermediate temperatures, the linear conductance behaves as $G \sim e^{-\epsilon_{0}(\phi) / T}$, in agreement with Eq. (39). This form of suppression resembles the thermal activation behavior $\left(G \sim e^{-\Delta / T}\right)$ in tunnel junctions [34]. At lower temperatures however, a deviation from such a behavior occurs. To examine this more carefully, and also to compare with the analytical results, we have plotted the same graph in logarithmic scale in Fig. 5b, adding to it the analytical curves (solid lines). Except for the $\phi=0$ case [where (39) vanishes], the agreement between the numerical and analytical results at intermediate temperatures is very good. At low $T$, on the other hand, the numerical curves show saturation. The crossover temperature to the saturation regime is proportional to $\epsilon_{0}(\phi)$ and is almost independent of $\omega$ (not shown in the figure).

Such a saturation does not occur in the analytical curves (naturally, was also not predicted in Ref. [32]), and is clearly a higher order property. As we mentioned before, the leading order result of (39) vanishes as $T \rightarrow 0$, therefore the only remaining contribution will be the higher order terms. To see this explicitly, in Fig. 6 we have plotted $G$ versus $\eta$ at a low frequency $(\omega=0.005)$ and for two different temperatures. One immediately notices significant difference in the $\eta$-dependence between the two cases. At high $T$ (Fig. 6b), the conductance follows $1 / \eta$ dependence in agreement with (39). At $T=0.01$ (Fig. 6a), on the other hand, all three curves show linear dependence on $\eta$, which is obviously higher order than $1 / \eta$. Physically, the residual conductance is a result of the overlap of the midgap states, broadened by finite $\eta$, at zero energy. Increasing $\eta$, increases the density of states at zero energy and therefore the conductance. In reality, $\eta$ is also temperature dependent and in $s$-wave superconductors, it vanishes at $T=0$, and so does $G$. (a)

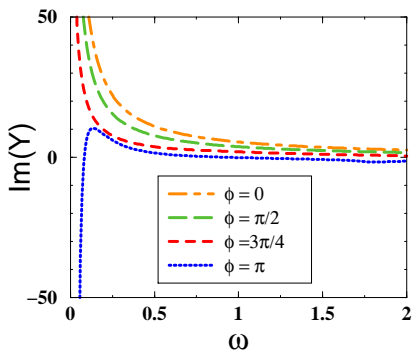

(b)

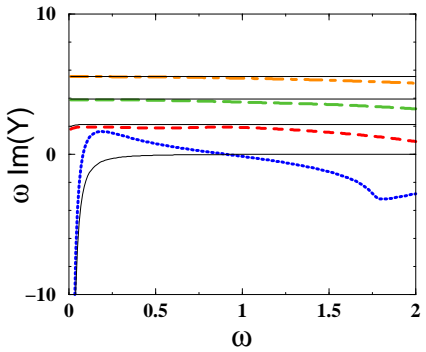

FIG. 7. (a) The imaginary part of the admittance as a function of $\omega$ at $T=0.1$. (b) The same data multiplied by $\omega$ together with the analytical (solid) curves.

Fig. 7a shows the frequency dependence of $\operatorname{Im}(Y)$ for different phase differences. Except for the $\phi=\pi$ curve, the other curves seem to show a $1 / \omega$ form (inductive behavior). To see this more clearly, we have plotted $\omega \operatorname{Im}(Y)$ as a function of $\omega$ in Fig. 7b. The first three curves are almost constant, confirming the $1 / \omega$ dependence. The curve at $\phi=\pi$, on the other hand, exhibits completely different behavior. In Fig. 7b, we also present the analytical curves corresponding to Eq. (38). The agreement between the numerical and analytical curves is good at low frequencies. At higher frequencies, all curves deviate from their analytical counterparts, as expected. Especially, for the case of $\phi=\pi$ the discrepancy between the two curves is significant.

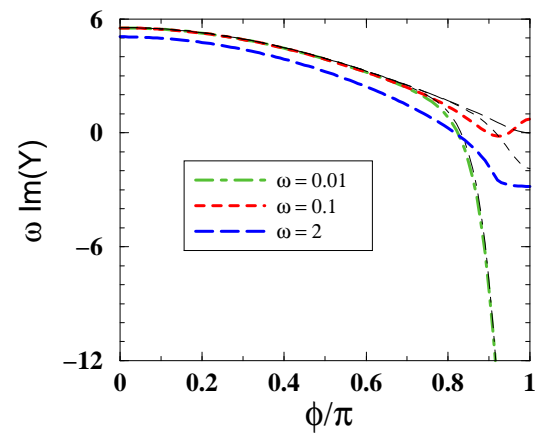

FIG. 8. $\omega \operatorname{Im}(Y)$ as a function of phase difference for different frequencies at $T=0.1$. The thin lines correspond to the low-frequency analytical results.

To understand this better, we have plotted $\omega \operatorname{Im}(Y)$ versus the phase difference $\phi$ for different frequencies in 
Fig. 8. The thin curves are plotted using Eq. (38). For $\omega=0.01$ and 0.1 , the curves overlap and agree quite well with the analytical ones over a wide range of $\phi$. Near $\phi=$ $\pi$, the curves separate and deviation from the analytical results become more evident. At high frequency $(\omega=2)$ on the other hand, the deviation already exists at $\phi=0$ and increases as $\phi \rightarrow \pi$.

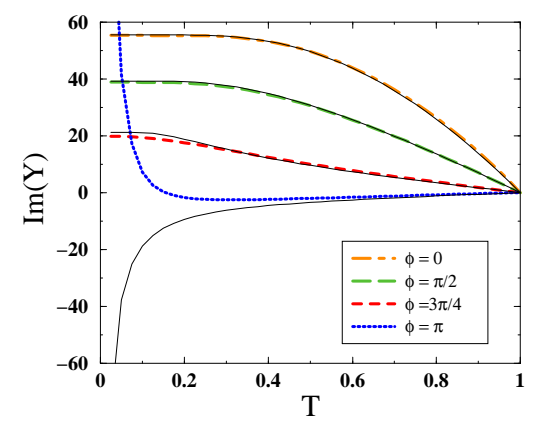

FIG. 9. $\operatorname{Im}(Y)$ as a function of temperature for different phase differences at $\omega=0.1$. The solid curves show the corresponding analytical results.

The temperature dependence of $\operatorname{Im}(Y)$, for different values of the phase difference, is plotted in Fig. 9. All the curves meet at $\operatorname{Im}(Y)=0$ as $T \rightarrow T_{c}$. The agreement with analytical results is very good at high $T$. At lower temperatures, the $\phi=\pi$ curve behaves completely differently than the other curves and deviates significantly from the analytical curve. This, as we mentioned before, is a result of the breakdown of the small frequency expansion at low temperatures.

\section{CONCLUSIONS}

We have presented a microscopic formalism for calculating ac properties of Josephson junctions. The method is based on linear response treatment of the non-equilibrium quasiclassical Green's function theory of superconductivity and uses a generalized form of the Riccati-transformations. The self-consistency equation for the linear response part of the order parameter, as well as the charge neutrality condition within the bulk, is satisfied to a very good approximation, with no need for numerical iteration.

We successfully applied the method to the case of a ballistic superconducting point contact and obtained nontrivial results for linear conductivity of the junction both analytically and numerically. We noticed strong temperature, frequency, and phase dependence in the real and imaginary parts of the ac conductivity. In particular, we found the conductance to be many orders of magnitude larger at $\phi=\pi$ than at smaller phase differences. This is a result of the influence of the zero energy bound states on the quasiparticle conductance. The agreement between the analytical and numerical results is very good at low frequencies. The exceptions happen near $\phi=0$ for $G$, and $\phi=\pi$ for $\operatorname{Im}(Y)$, where the leading order contributions vanish or become comparable to the neglected terms. The discrepancy becomes more pronounced at low $T$ or high $\omega$, where the validity of the leading order approximation become questionable.

Experimentally, superconducting point contacts have been realized using techniques such as: scanning tunneling microscopy [44], mechanically controllable break junctions $[45,46]$, superconductor-2 dimensional electron gas-superconductor junctions $[47,48]$, etc. Subgap structures were observed [49] and nice measurements of transmission coefficients of individual quantum channels were performed by Scheer et al. [50]. Unfortunately, phasedependent measurement of the conductance is difficult and the only available data (to our knowledge) is those by Rifkin and Deaver [51]. They found a strongly phase dependent conductance, in qualitative agreement with our results. More experimental research is necessary to confirm the predictions of the present work.

In this article, we have only considered a contact with perfect transparency. The method, however, is general and applicable for arbitrary transparency. One only needs to take into account appropriate (e.g. Zaitsev) boundary conditions at the contact. A solution to the Zaitsev boundary conditions, suitable for the present calculation method, is given in Ref. [22]. The method proposed in this article is also applicable to other systems such as grain boundary junctions between unconventional superconductors, which is the subject of a separate publication [52].

\section{ACKNOWLEDGEMENT}

The author has greatly benefited from discussion with A. Leggett, A. Omelyanchouk, A. Smirnov, A. Zagoskin, and especially A. Maassen van den Brink because of helpful suggestions in the preparation of the manuscript and providing Eq. (A14) as the appropriate form for the gauge transformation.

\section{APPENDIX A: QUASICLASSICAL KELDYSH GREEN'S FUNCTIONS}

The quasiclassical Green's function [43] $\breve{g}\left(\mathbf{v}_{F}, \epsilon ; \mathbf{r}, t\right)$ is a $2 \times 2$ matrix, every element of which also a $2 \times 2$ matrix, and a function of the Fermi velocity $\mathbf{v}_{F}$, quasiparticle energy $\epsilon$, position $\mathbf{r}$, and time $t$. We represent $\breve{g}$ as

$$
\breve{g}=\left(\begin{array}{cc}
\widehat{g}^{R} & \widehat{g}^{K} \\
0 & \widehat{g}^{A}
\end{array}\right),
$$

where the matrices $\widehat{g}^{R}, \widehat{g}^{A}, \widehat{g}^{K}$ are the quasiclassical retarded, advanced, and Keldysh Green's functions in Nambu-Gorkov representation, respectively: 


$$
\widehat{g}^{R, A}=\left(\begin{array}{cc}
g^{R, A} & f^{R, A} \\
f^{R, A \dagger} & -g^{R, A \dagger}
\end{array}\right), \quad \widehat{g}^{K}=\left(\begin{array}{cc}
g^{K} & f^{K} \\
-f^{K \dagger} & g^{K \dagger}
\end{array}\right) .
$$

The $\dagger$-operation performs the following transformation

$$
\mathcal{O}^{\dagger}\left(\mathbf{v}_{F}, \epsilon ; \mathbf{r}, t\right)=\mathcal{O}\left(-\mathbf{v}_{F},-\epsilon ; \mathbf{r}, t\right)^{*} .
$$

In frequency domain it also changes $\omega$ to $-\omega$. The retarded and advanced Green's functions carry information about the energy spectrum of the electronic states of the system, while the Keldysh ones have information about occupation of those states.

The following symmetries hold for the Green's functions:

$$
g^{A}=-g^{R *}, \quad f^{R \dagger}=f^{A *}
$$

and

$$
g^{K}=g^{K *}, \quad f^{K \dagger}=f^{K *} .
$$

In frequency space, these give $g^{A}(\omega)=-g^{R}(-\omega)^{*}$, etc.

The equation of motion that describes the time evolution of $\breve{g}$ is written as

$\mathbf{v}_{F} \cdot \nabla \breve{g}-\left[i\left(\epsilon-\frac{e}{c} \mathbf{v}_{F} \cdot \mathbf{A}\right) \breve{\tau}_{3}-\breve{\Delta}+i e \Phi \breve{1}, \breve{g}\right]_{\otimes}=\breve{0}$

accompanied by the normalization condition

$$
\breve{g} \otimes \breve{g}=\breve{1},
$$

where $\Phi$ and $\mathbf{A}$ are the scalar and vector potentials respectively, and $[A, B]_{\otimes} \equiv A \otimes B-B \otimes A$, with

$$
(A \otimes B)(\epsilon, t)=e^{\frac{i}{2}\left(\partial_{\epsilon}^{A} \partial_{t}^{B}-\partial_{t}^{A} \partial_{\epsilon}^{B}\right)} A(\epsilon, t) B(\epsilon, t) .
$$

The product $(\mathrm{A} 8)$ is associative and satisfies $(A \otimes B)^{\dagger}=$ $A^{\dagger} \otimes B^{\dagger}$, but $(A \otimes B)^{*}=B^{*} \otimes A^{*}$. We also have

$$
\breve{\tau}_{3}=\left(\begin{array}{cc}
\widehat{\tau}_{3} & 0 \\
0 & \widehat{\tau}_{3}
\end{array}\right), \quad \breve{\Delta}=\left(\begin{array}{cc}
\widehat{\Delta} & 0 \\
0 & \widehat{\Delta}
\end{array}\right),
$$

where the $2 \times 2$ Pauli matrix $\widehat{\tau}_{3}$ and $\widehat{\Delta}$ are

$$
\widehat{\tau}_{3}=\left(\begin{array}{cc}
1 & 0 \\
0 & -1
\end{array}\right), \quad \widehat{\Delta}=\left(\begin{array}{cc}
0 & \Delta \\
\Delta^{\dagger} & 0
\end{array}\right),
$$

with $\Delta$ being the superconducting pairing potential. The constant $e$ in (A6) is the absolute value of the electronic charge, and $c$ is the speed of light. In equilibrium, Eq. (A6) reduces to the Eilenberger equation (see Ref. [13] for example), by changing $\epsilon \rightarrow i \omega_{n}$, where $\omega_{n}$ are the Matsubara frequencies.

A gauge transformation is defined by the following simultaneous operations:

$$
\begin{aligned}
\breve{\Delta} & \mapsto e^{i(\chi / 2) \breve{\tau}_{3}} \breve{\Delta} e^{-i(\chi / 2) \breve{\tau}_{3}}, \\
\Phi & \mapsto \Phi+\frac{1}{2 e} \partial_{t} \chi, \\
\mathbf{A} & \mapsto \mathbf{A}-\frac{c}{2 e} \nabla \chi, \\
\breve{g} & \mapsto e^{i(\chi / 2) \breve{\tau}_{3}} \otimes \breve{g} \otimes e^{-i(\chi / 2) \breve{\tau}_{3}} .
\end{aligned}
$$

It therefore takes the phase of the order parameter $\phi$ to $\phi+\chi$. In the discussion of section II, we have chosen $\chi=-\delta \phi$.

The Green's functions can be used to calculate physical quantities. The quasiparticle density of states is given by

$$
N(\epsilon)=\frac{N_{F}}{4}\left\langle\operatorname{Tr}\left[\widehat{\tau}_{3}\left(\widehat{g}^{R}-\widehat{g}^{A}\right)\right]\right\rangle,
$$

where $N_{F}$ is the density of state of electrons at the Fermi surface and $\langle\ldots\rangle$ denotes averaging over $\mathbf{v}_{F}$. The pairing potential satisfies the following self-consistency relation:

$$
\widehat{\Delta}\left(\mathbf{v}_{F}\right)=\frac{N_{F}}{4 i} \int_{-\epsilon_{c}}^{\epsilon_{c}} d \epsilon\left\langle V\left(\mathbf{v}_{F}, \mathbf{v}_{F}^{\prime}\right) \widehat{f}^{K}\left(\mathbf{v}_{F}^{\prime}\right)\right\rangle_{\mathbf{v}_{F}^{\prime}},
$$

where $\widehat{f}^{K}$ is the off-diagonal part of $\widehat{g}^{K}$, and $V\left(\mathbf{v}_{F}, \mathbf{v}_{F}^{\prime}\right)$ is the interaction potential. Furthermore, the current density is given by

$$
\mathbf{j}=\frac{e N_{F}}{4} \int_{-\epsilon_{c}}^{\epsilon_{c}} d \epsilon\left\langle\mathbf{v}_{F} \operatorname{Tr}\left[\widehat{\tau}_{3} \widehat{g}^{K}\right]\right\rangle
$$

and the charge density by

$$
\rho=e N_{F}\left(-2 e \Phi+\frac{1}{4} \int_{-\epsilon_{c}}^{\epsilon_{c}} d \epsilon\left\langle\operatorname{Tr}\left[\widehat{g}^{K}\right]\right\rangle\right) .
$$

\section{APPENDIX B: GENERALIZED RICCATI-TRANSFORMATION}

Riccati-transformations [20] are proven to be very useful tools for numerical calculations of equilibrium properties of superconducting systems. For non-equilibrium systems, however, the presence of the $\otimes$-operators makes the formalism nontrivial. Nevertheless, a generalization of the standard transformation to the non-equilibrium case is possible. It is common to define Riccati amplitudes $a^{\alpha}$ and $b^{\alpha}$, where $\alpha=R, A$ for the retarded and advanced Green's functions respectively. They are related to the corresponding Green's functions by $[21,22]$

$$
\begin{aligned}
& g^{\alpha}=s^{\alpha}\left(1+a^{\alpha} \otimes b^{\alpha}\right)^{-1} \otimes\left(1-a^{\alpha} \otimes b^{\alpha}\right), \\
& f^{\alpha}=s^{\alpha}\left(1+a^{\alpha} \otimes b^{\alpha}\right)^{-1} \otimes\left(2 a^{\alpha}\right),
\end{aligned}
$$

where $s^{\alpha}$ is defined in Eq. (5). Here we define the inverse operation by $A^{-1} \otimes A=A \otimes A^{-1}=1$. Eqs. (B1) already resemble their counterparts in the standard Matsubara formalism [20]. It is straightforward to show that for $\mathbf{A}=$ 0 these functions satisfy Riccati-type equations given by 


$$
\begin{aligned}
\mathbf{v}_{F} \cdot \nabla a^{\alpha} & =2 i \epsilon a^{\alpha}-a^{\alpha} \otimes \Delta^{\dagger} \otimes a^{\alpha}+\Delta+\left[i e \Phi, a^{\alpha}\right]_{\otimes}, \\
-\mathbf{v}_{F} \cdot \nabla b^{\alpha} & =2 i \epsilon b^{\alpha}-b^{\alpha} \otimes \Delta \otimes b^{\alpha}+\Delta^{\dagger}-\left[i e \Phi, b^{\alpha}\right]_{\otimes}
\end{aligned}
$$

In equilibrium, the $\otimes$-operation is replaced by a simple multiplication and these equations reduce to (6).

It is also necessary to define other functions $a^{K}$ and $b^{K}$ [53], which are related only to the Keldysh Green's functions $[21,22]$

$$
\begin{aligned}
g^{K}= & 2\left(1+a^{R} \otimes b^{R}\right)^{-1} \otimes\left(a^{K}+a^{R} \otimes b^{K} \otimes b^{A}\right) \\
& \otimes\left(1+a^{A} \otimes b^{A}\right)^{-1}, \\
f^{K}= & 2\left(1+a^{R} \otimes b^{R}\right)^{-1} \otimes\left(a^{K} \otimes a^{A}-a^{R} \otimes b^{K}\right) \\
& \otimes\left(1+b^{A} \otimes a^{A}\right)^{-1},
\end{aligned}
$$

and are governed by the following dynamical equations

$$
\begin{aligned}
\mathbf{v}_{F} \cdot \nabla a^{K}= & -\partial_{t} a^{K}-a^{R} \otimes \Delta^{\dagger} \otimes a^{K}+a^{K} \otimes \Delta \otimes b^{A} \\
& +\left[i e \Phi, a^{K}\right]_{\otimes}, \\
-\mathbf{v}_{F} \cdot \nabla b^{K}= & -\partial_{t} b^{K}-b^{R} \otimes \Delta \otimes b^{K}+b^{K} \otimes \Delta^{\dagger} \otimes a^{A} \\
& -\left[i e \Phi, b^{K}\right]_{\otimes} .
\end{aligned}
$$

The functions $a^{\alpha}$ and $b^{\alpha}$ are related, by the $\dagger$-operation [Eq. (A3)], through $b^{\alpha}=a^{\alpha \dagger}$ for $\alpha=R, A, K$. In addition, the symmetries (A4) and (A5) require

$$
a^{A}=-b^{R *}, \quad a^{K}=a^{K *} .
$$

One can show that Eqs. (B1)-(B4) satisfy the dynamical equation (A6) together with the normalization condition (A7).

In equilibrium, Eqs. (B1) reduce to (4), and (B3) give

$$
\begin{aligned}
g_{0}^{K} & =2 \frac{a_{0}^{K}+a_{0}^{R} b_{0}^{K} b_{0}^{A}}{\left(1+a_{0}^{R} b_{0}^{R}\right)\left(1+a_{0}^{A} b_{0}^{A}\right)}, \\
f_{0}^{K} & =2 \frac{a_{0}^{K} a_{0}^{A}-a_{0}^{R} b_{0}^{K}}{\left(1+a_{0}^{R} b_{0}^{R}\right)\left(1+a_{0}^{A} b_{0}^{A}\right)} .
\end{aligned}
$$

Satisfying (9), one finds

$$
a_{0}^{K}=\left(1+a_{0}^{R} b_{0}^{A}\right) \mathcal{F}, \quad b_{0}^{K}=-\left(1+a_{0}^{A} b_{0}^{R}\right) \mathcal{F} .
$$

Linear response equations (13) and (14) are obtained by expanding (B1) to the linear order. To obtain (16) and (17), we expand (B3) to the linear order and introduce

$$
\delta \widehat{g}^{X}=\left(\begin{array}{cc}
\delta g^{X} & \delta f^{X} \\
\delta f^{X \dagger} & -\delta g^{X \dagger}
\end{array}\right)
$$

through (15). We also define $\delta a^{X}$ and $\delta b^{X}$ in terms of the linear response $\delta a^{K}$ and $\delta b^{K}$ by

$$
\begin{aligned}
\delta a^{K} & =\delta a^{X}\left(\mathcal{F}_{+}-\mathcal{F}_{-}\right)+\delta a^{R} b_{0-}^{A} \mathcal{F}_{-}+\delta b^{A} a_{0+}^{R} \mathcal{F}_{+}, \\
\delta b^{K} & =\delta b^{X}\left(\mathcal{F}_{-}-\mathcal{F}_{+}\right)-\delta b^{R} a_{0-}^{A} \mathcal{F}_{-}-\delta a^{A} b_{0+}^{R} \mathcal{F}_{+} .
\end{aligned}
$$

The differential equations (18) then follow directly from (B2) and (B4). It should finally be mentioned that the $A$ 's and B's in Eq. (18) are related by: $\widetilde{A}^{\alpha}=A^{\alpha \dagger}, \widetilde{B}^{\alpha}=$ $B^{\alpha \dagger}$.
[1] D. Xu, S.K. Yip, and J.A. Sauls, Phys. Rev. B 51, 16233 (1995).

[2] N. Schopohl and K. Maki, Phys. Rev. B 52, 490 (1995).

[3] M. Ichioka, N. Hayashi, N. Enomoto, and K. Machida, J. Phys. Soc. Jpn. 64, 4547 (1995).

[4] D. Rainer, J.A. Sauls, and D. Waxman, Phys. Rev. B 54, 10094 (1996).

[5] M. Matsumoto and H. Shiba, J. Phys. Soc. Jpn. 64, 3384 (1995); 64, 4867 (1995); 65, 2192 (1996).

[6] L.J. Buchholtz, M. Palumbo, D. Rainer, and J.A. Sauls, J. Low Temp. Phys. 101, 1079 (1995); 101, 1099 (1995); M. Fogelström, D. Rainer, J.A. Sauls, Phys. Rev. Lett. 79, 281 (1997).

[7] I.O. Kulik and A.N. Omelyanchouk, Sov. J. Low Temp. Phys. 3, 459 (1977); 4, 142 (1978).

[8] A.V. Zaitsev, Sov. Phys. JETP 51, 111 (1980).

[9] I.O. Kulik and A.N. Omelyanchouk, Sov. J. Low Temp. Phys. 9, 189 (1983).

[10] J.C. Cuevas, A. Martín-Rodero, and A.L. Yeyati, Phys. Rev. B 54, 7366 (1996); A. Martín-Rodero, A. Levy Yeyati, and F.J. Garca-Vidal, Phys. Rev. B 53, R8891 (1996).

[11] Yu.S. Barash, A.V. Galaktionov, and A.D. Zaikin, Phys. Rev. B 52, 665 (1995).

[12] S. Yip, Phys. Rev. B 52, 3087 (1995); M. Fogelström and S.K. Yip, Phys. Rev. B 57, R14060 (1998).

[13] M.H.S. Amin, A.N. Omelyanchouk, and A.M. Zagoskin, Phys. Rev. B 63, 212502 (2000); M.H.S. Amin, A.N. Omelyanchouk, S.N. Rashkeev, M. Coury, and A.M. Zagoskin, Physica B 318, 162 (2002).

[14] M.H.S. Amin, S.N. Rashkeev, M. Coury, A.N. Omelyanchouk, and A.M. Zagoskin, Phys. Rev. B 66, 174515 (2002).

[15] G. Eilenberger, Z. Phys. 214, 195 (1968).

[16] A.I. Larkin and N.Y. Ovchinnikov, Zh. Éksp. Theor. Fiz. 55, 2262 (1968) [Sov. Phys. JETP 28, 1200 (1969)].

[17] A.A. Abrikosov, L.P. Gorkov, and I.E. Dzyaloshinski, "Methods of Quantum Field Theory in Statistical Physics", Prentice-Hall Inc. (1963).

[18] G.M. Eliashberg, Zh. Éksp. Theor. Fiz. 61, 1254 (1973) [Sov. Phys. JETP 34, 668 (1973)].

[19] A.I. Larkin and N.Y. Ovchinnikov, Zh. Éksp. Theor. Fiz. 68, 1915 (1975); 73, 299 (1977) [Sov. Phys. JETP 41, 960 (1975); 46, 155 (1977)].

[20] N. Schopohl, in Quasiclassical Theory of Superconductivity, Proceedings of the Workshop Quasiclassical Methods of Superconductivity, Verditz, Autria, 1996, edited by D. Rainer amd J.A. Sauls (1998), p. 88.

[21] M. Eschrig, J.A. Sauls, and D. Rainer, Phys. Rev. B 60, 10447 (1999).

[22] M. Eschrig, Phys. Rev. B 61, 9061 (2000).

[23] see for example: J.R. Kirtley, Physica C 368, 55 (2002), and references therein.

[24] M.H.S. Amin, A.N. Omelyanchouk, and A.M. Zagoskin, Physica C 372-376 P1, 184 (2002); M.H.S. Amin, M. Coury, and G. Rose, IEEE Trans. Appl. Supercond. 12, 1877 (2002).

[25] K.K. Likharev, in "Applications of superconductivity", edited by H. Weinstock, Kluwer, Dordrecht (2000).

[26] Y. Makhlin, G. Schön, and A. Shnirman, Rev. Mod. 
Phys. 73, 357 (2001).

[27] M.H.S. Amin, A.N. Omelyanchouk, A. Blais, A. Maassen van den Brink, G. Rose, T. Duty, and A.M. Zagoskin, Physica C 368, 310 (2002).

[28] K.K. Likharev, Rev. Mod. Phys. 51, 101 (1979).

[29] U. Gunsenheimer and A.D. Zaikin, Phys. Rev. B 50, 6317 (1994).

[30] E.N. Bratus, V.S. Shumeiko, and G. Wendin, Phys. Rev. Lett. 74, 2110 (1995); M. Hurd, T. Löfwander, G. Johansson, and G. Wendin, Phys. Rev. B 59, 4412 (1999).

[31] D. Averin and A. Bardas, Phys. Rev. Lett. 75, 1831 (1995); A. Bardas and D.V. Averin, Phys. Rev. B. 56, R8518 (1996).

[32] D. Averin and A. Bardas, Phys. Rev. B. 53, R1705 (1996).

[33] A.V. Zaitsev and D.V. Averin, Phys. Rev. Lett. 80, 3602 (1998).

[34] M. Tinkham, "Introduction to Superconductivity", 2nd ed. McGraw-Hill, New York, (1996).

[35] T.M. Klapwijk, G.E. Blonder, and M. Tinkham, Physica B 109\&110, 1657 (1982).

[36] A.M. Zagoskin, "Quantum Theory of Many-Body Systems", Springer-Verlag, New York (1998).

[37] U. Eckern, G. Schön, and V. Ambegaokar, Phys. Rev. B 30, 6419 (1984).

[38] In general, a large voltage applied to a Josephson junction may affect the modulus of the order parameter. This however is not a concern in the linear response limit.

[39] In the superconducting point contact considered here, $j_{\mathrm{c}}(r) / j_{\mathrm{c}, \text { bulk }} \sim(d / r)^{2}$, where $r\left(\sim \xi_{0} \gg d\right)$ is the distance from the orifice and $d$ is the diameter of the hole (see Fig. 1).

[40] Here we include quasiparticle damping phenomenologically by introducing $\eta$. A more rigorous calculation should take into account electron-phonon interaction directly, as the main cause for inelastic scattering.

[41] In this article we only consider singlet superconductors in which $\Delta\left(-\mathbf{v}_{F}\right)=\Delta\left(\mathbf{v}_{F}\right)$, and therefore in equilibrium $\Delta_{0}^{\dagger}=\Delta_{0}^{*}$. For the linear response $\delta \Delta$, it follows directly from (3) and (A3) that $\delta \Delta^{\dagger}(\omega)=-\delta \Delta^{*}(\omega)$, if we take $\Phi(t)$ to be real and $\Phi(-\omega)=\Phi(\omega)$. The latter is just for convenience; other choices will give the same impedance. To obtain general relations, one should replace $\Delta_{0}^{*}$ and $\delta \Delta^{*}$ with $\Delta_{0}^{\dagger}$ and $-\delta \Delta^{\dagger}$ respectively.

[42] In order to obtain Eq. (7) of Ref. [32], one should replace $2 \eta$ with $\gamma\left(\epsilon_{0}\right)$.

[43] J. Rammer and H. Smith, Rev. Mod. Phys. 58, 323 (1988).

[44] N. Agraït, J. G. Rodrigo, and S. Vieira, Phys. Rev. B, 47, 12345, (1993).

[45] C.J. Muller, J.M. Ruitenbeek, and L.J. de Jong, Physica C 191, 485 (1992).

[46] B.J. Vleeming, C.J. Mullerm M.C. Koops, and R. de Bruyan Ouboter, Phys. Rev. B, 50, 16741, (1994).

[47] H. Takayanagi, T. Akazaki, and J. Nita, Phys. Rev. Lett. 75, 3533 (1995);

[48] L.C. Mur, C.J.P.M. Harmans, J. E. Mooij, J. F. Carlin, A. Rudra, and M. Ilegems, Phys. Rev. B 54, R2327 (1996).

[49] A. W. Kleinsasser, R. E.Miller, W.H. Mallison, and G.
B. Arnold, Phys. Rev. Lett. 72, 1738 (1994); N. van der Post, E.T. Peters, I.K. Yanson, J.M. van Ruitenbeek, Phys. Rev. Lett. 73, 2611 (1994); B.J. Vleeming, et al., Phys. Rev. B 50, 16741 (1994).

[50] E. Scheer, P. Joyez, D. Esteve, C. Urbina, and M.H. Devoret, Phys. Rev. Lett. 78, 3535 (1997); E. Scheer, N. Agraï, J.C. Cuevas, A. Levy Yeyati, B. Ludoph, A. Martín-Rodero, G. Rubio Bullinger, J.M. van Ruitenbeek, and C. Urbina, Nature 394, 154 (1998).

[51] R. Rifkin and B.S. Deaver, Jr., Phys. Rev. B 13, 3894 (1976).

[52] M.H.S. Amin and A.Yu. Smirnov, preprint (condmat/0304255).

[53] These are indeed equivalent to the distribution functions $x^{K}$ and $\widetilde{x}^{K}$ defined in Refs. [21,22]. 